\title{
FREE ENERGY AS A GEOMETRIC INVARIANT
}

\author{
MARK POLLICOTT AND HOWARD WEISS
}

The University of Manchester and The Pennsylvania State University

February 27, 2005 2:44pm

\begin{abstract}
The free energy plays a fundamental role in statistical and condensed matter physics. A related notion of free energy plays an important role in the study of hyperbolic dynamical systems. In this paper we introduce and study a natural notion of free energy for surfaces with variable negative curvature. This geometric free energy encodes a new type of marked length spectrum of closed geodesics, which lies between the well-known marked length spectrum (marked by the corresponding element of the fundamental group) and the unmarked length spectrum. We prove that the free energy parametrizes the boundary of the domain of convergence of a Poincaré series which also encodes this spectrum. We also show that this new length spectrum, or equivalently the geometric free energy, is not an isometry invariant. In the final section we use tools from multifractal analysis to effect a fine asymptotic comparison of word length and geodesic length of closed geodesics.

We hope that our geometric understanding of free energy will provide new insight into this fundamental physical and dynamical quantity.
\end{abstract}

\section{INTRODUCTION}

The free energy, which can be viewed as a generating function for the sequence of energy levels of a system, plays a fundamental role in statistical and condensed matter physics. A related notion of free energy plays an important role in hyperbolic dynamical systems, where it can be viewed as a generating function for the orbital averages of a function over the closed orbits. In this paper we introduce and study a natural notion of free energy for surfaces with variable negative curvature.

The geometric free energy is defined as a generating function for the lengths of closed geodesics indexed by the lengths of corresponding words in the fundamental group, as follows. Let $V$ be a compact negatively curved surface and let $\Gamma$ denote a set of generators for the fundamental group $\pi_{1}(V)$ of $V$. The symbol $\gamma$ will always denote a closed geodesic,

Key words and phrases. Pressure, free energy, thermodynamic formalism, length spectrum, negatively curved surface, word length, Poincaré series, multifractal analysis.

The work of the second author was partially supported by a National Science Foundation grant DMS0100252. This work was completed during a visit by the first author to Penn State as a Shapiro Fellow.

Typeset by $\mathcal{A M S}_{\mathcal{M}}-\mathrm{T}_{\mathrm{EX}}$ 
$[\gamma]$ the free homotopy class of $\gamma$ in $\pi_{1}(V)$, and $l(\gamma)$ the geodesic length of $\gamma$. Every free homotopy class contains a unique closed geodesic. We let $|\gamma|$ denote the word length of the free homotopy class of $[\gamma]$, i.e., where $|\gamma|$ the smallest number of generators from $\Gamma$ needed to represent an element in $[\gamma]$. We define the free energy of $\mathbf{V}$ by

$$
F(t)=F_{\Gamma, V}(t) \stackrel{\circ}{=} \lim _{n \rightarrow+\infty} \frac{1}{n} \log \sum_{\substack{[\gamma] \in \pi_{1}(V) \\|\gamma|=n}} \exp (-t l(\gamma)) .
$$

This definition arises as a special case of the dynamical free energy for the geodesic flow, where the geodesic flow is coded by the geometric Bowen-Series Markov partition. This is carefully explained in Section 1. We also prove that the free energy parametrizes the boundary of the domain of convergence of the related Poincaré series

$$
\sum_{[\gamma] \in \pi_{1}(V)} \exp (-a l(\gamma)-b|\gamma|)
$$

There is extensive literature on the rigidity properties of the marked and unmarked length spectrum of closed geodesics on a negatively curved surface. The marked length spectrum consists of the lengths of closed geodesics marked by the corresponding free homotopy class of the geodesic, i.e., the sequence of pairs $\left\{([\gamma], l(\gamma)), \gamma \in \pi_{1}(V)\right\}$. The marked length spectrum is an isometry invariant [Cro, Ota]. The unmarked length spectrum is the sequence consisting of just the lengths of the closed geodesics and is known not to be an isometry invariant [Bus, Mck, Sun, Vig, Wol]. Our geometric free energy encodes a third type of marked length spectrum of closed geodesics given by the sequence of pairs $\left\{(|\gamma|, l(\gamma)), \gamma \in \pi_{1}(V)\right\}$, which lies between the marked length spectrum and the unmarked length spectra. The relationship between the word length and geometric length of closed geodesics was first studied by Milnor [Mil]. We show that this length spectrum, or equivalently the geometric free energy, is not an isometry invariant.

In the final section we use tools from multifractal analysis to effect a fine asymptotic comparison of word length and geodesic length of closed geodesics. We hope that our geometric understanding of free energy will provide new insight into this fundamental physical and dynamical quantity.

This note can be viewed as a continuation of $[\mathrm{PW}$, where the authors study the free energy of a classical one-dimensional lattice spin system as a dynamical invariant. All of the facts from thermodynamic formalism we require can be found in [Rue] and [PP].

\section{Free Energy and Poincaré series.}

Let $\phi^{t}: T_{1} V \rightarrow T_{1} V$ denote the geodesic flow on the unit tangent bundle of $V$. Our approach starts with the Bowen-Series coding [BS, TSK] to obtain a symbolic representation of the geodesic flow on $T_{1} V$. While there are other methods of constructing Markov partitions for the geodesic flow [Bow, Rat, Sin], this method, which codes intersections of geodesics with sides of a fundamental polygon, is the only known coding scheme which 
faithfully codes the lengths and combinatorics of (essentially) all closed geodesics. It produces a suspension flow over a subshift of finite type with a roof function whose Birkhoff sum over a periodic orbit of period $n$ is precisely the length of the corresponding closed geodesic, where $n$ is also the word length in $\pi_{1}(V)$ of the closed geodesic.

The Bowen-Series coding starts with a specially chosen fundamental polygon for the fundamental group $\Gamma$ of $V$ in the universal covering surface. Each side of the finite-sided polygon is a subarc of a closed geodesic corresponding to some element of $\Gamma$. One then identifies vaiours pairs of the geodesic subarcs using covering transformations, and verifies that the restrictions of these identification maps to the boundary of the universal covering (which can be identified with the unit circle) determines a Markov map $T$ of the unit circle. By construction, $T$ is orbit equivalent to the action of $\Gamma$ on the boundary of the universal covering.

The Markov map $T$ is naturally coded by a subshift of finite type $\sigma: \Sigma_{A}^{+} \rightarrow \Sigma_{A}^{+}$. There is also a natural Hölder continuous roof function $r: \Sigma_{A}^{+} \rightarrow \mathbb{R}$ defined by the time required for points on the special geodesic arcs to intersect other special geodesic arcs under the geodesic flow. One then defines the suspension space $X_{r}=\Sigma_{A}^{+} \times \mathbb{R} / \mathbb{Z}$, where $\mathbb{Z}$ is the group of maps generated by $(x, y) \rightarrow(\sigma x, y-r(x))$, and the suspension flow $\sigma_{r}^{t}: X_{r} \rightarrow X_{r}$ induced by the maps $(x, t) \rightarrow(x, y+t)[\mathrm{PP}]$. The geodesic flow $\phi^{t}$ and the suspension flow are related by the existence of a bounded-to-one continuous surjection $p: X_{r} \rightarrow T_{1} V$ such that $\phi^{t} \circ p=\pi \circ \sigma_{r}^{t}$.

Moreover, given two negatively curved metrics on the same topological surface, the underlying subshifts of finite type $\sigma: \Sigma_{A}^{+} \rightarrow \Sigma_{A}^{+}$from the Bowen-Series coding of their geodesic flows are the same. Finally, every closed geodesic $\gamma$ corresponds to a periodic orbit $\left\{x, \sigma x, \ldots, \sigma^{n-1} x\right\}$ of $\Sigma_{A}^{+}$with $\sigma^{n} x=x$, where the Birkhoff sum $S_{n} r(x)=\sum_{k=0}^{n-1} r\left(\sigma^{k} x\right)=$ $l(\gamma)$ and $n=|\gamma|$. This correspondence is one-to-one, except for an at most finite set of exceptional prime geodesics, which have no effect on our results.

These special properties of the Bowen-Series coding are crucial for our analysis. If one could construct a symbolic coding of the geodesic flow for any Riemannian metric satisfying these properties, then all the results in this paper would immediately carry through to this metric. In $[\mathrm{AR}]$ the authors state technical conditions for a more general Markov partition to possess these properties, but there are very few examples in dimensions greater than two which have been shown to satisfy these properties.

Using the Bowen-Series coding, we define the free energy of the negatively curved surface $V$ to be the free energy of the roof function $r: \Sigma_{A}^{+} \rightarrow \mathbb{R}$, i.e.,

$$
F(t)=F_{\Gamma, V}(t) \stackrel{\circ}{=}(-t r)
$$

where $P$ denotes the thermodynamic pressure defined on all real valued continuous functions on $\Sigma_{A}^{+}$. Using the periodic orbit definition of pressure $[\mathrm{PP}]$ we have

$$
P(-t r)=\lim _{n \rightarrow+\infty} \frac{1}{n} \log \sum_{\sigma^{n} x=x} \exp \left(-t S_{n} r(x)\right) .
$$


It immediately follows from faithful coding properties of closed geodesics for the BowenSeries coding that the free energy of $V$ has the following geometric realization, which we define to be the free energy of $\mathbf{V}$

$$
F(t)=F_{\Gamma, V}(t) \stackrel{\circ}{=} \lim _{n \rightarrow+\infty} \frac{1}{n} \log \sum_{\substack{[\gamma] \in \pi_{1}(V) \\|\gamma|=n}} \exp (-t l(\gamma)) .
$$

It follows from standard facts in thermodynamic formalism [PP, Rue] that this free energy is a smooth and strictly convex function.

We now define a modified Poincaré series for $V$ and $\Gamma$ by

$$
\rho(a, b)=\rho_{\Gamma, V}(a, b) \stackrel{\circ}{=} \sum_{[\gamma] \in \pi_{1}(V)} \exp (-a l(\gamma)-b|\gamma|) .
$$

Our next goal is to show that the free energy parametrizes the boundary curve separating the domain of convergence of this Poincaré series from the domain of divergence.

We recall that by a classical result of Milnor [Mil], there exist $A, B>0$ such that $A \leq l(\gamma) /|\gamma| \leq B$ for all closed geodesics $\gamma$. If we choose $A=\inf _{\gamma} l(\gamma) /|\gamma|$ and $B=$ $\sup _{\gamma} l(\gamma) /|\gamma|$ then using the Anosov closing lemma, it is easy to show that the ratios $\{l(\gamma) /|\gamma|: \gamma=$ closed geodesic $\}$ are dense in the interval $[A, B]$.

It follows from Milnor's result that the Poincaré series $\rho(a, b)$ converges provided $a, b>0$ are sufficiently large. We denote by

$$
\mathcal{R}=\mathcal{R}(\Gamma, V)=\left\{(a, b) \in \mathbb{R}^{2}: \rho(a, b)<+\infty\right\}
$$

the domain of convergence of the Poincare series and $\mathcal{L}=\mathcal{L}(\Gamma, V)$ the boundary curve of $\mathcal{R}$ (see Figure 1). The following proposition provides the important link between the free energy and the Poincaré series.

Proposition 1. The free energy $F$ parametrizes the boundary curve $\mathcal{L}$ in the sense that $\mathcal{L}=\{(a, F(a)\}$ for $-\infty<a<+\infty$.

Proof. Using the key property of the Bowen-Series coding we can rewrite the Poincaré series in the following form

$$
\rho(a, b)=\rho_{\Gamma, V}(a, b) \stackrel{\circ}{=} \sum_{n=1}^{\infty} \frac{1}{n} \sum_{\sigma^{n} x=x} \exp \left(-a S_{n} r(x)-b n\right) .
$$

By the root test, this infinite series converges if

$$
\exp (P(-a r-b))=\lim _{n \rightarrow+\infty}\left(\sum_{\substack{\sigma^{n} x=x \\ 4}} \exp \left(-a S_{n} r(x)-b n\right)\right)^{1 / n}<1
$$


In particular, we immediately see that the sets $\mathcal{L}$ and $\mathcal{R}$ have the following simple interpretation in terms of symbolic dynamics

$$
\mathcal{R}=\left\{(a, b) \in \mathbb{R}^{2}: P(-a r-b)<0\right\}
$$

and

$$
\mathcal{L}=\left\{(a, b) \in \mathbb{R}^{2}: P(-a r-b)=0\right\} .
$$

Since the pressure $P(-a r-b)=0$ for $(a, b) \in \mathcal{L}$, we see that $b=P(-a r)=F(a)$.

We now define the generating function for word length in $\pi_{1}(V)$ by

$$
G(z)=\sum_{n=1}^{\infty} z^{n} \operatorname{card}\{\gamma:|\gamma|=n\}
$$

This is well known to be a rational function [Can, Eps]. The next proposition states that $\mathcal{L}$ is a smooth curve and identifies some special values on $\mathcal{L}$.

\section{Proposition 2.}

(a) The curve $\mathcal{L}$ is real analytic and strictly convex.

(b) The points $(0,1)$ and $(h, 0)$ lie on $L$, where

$$
h=\lim _{n \rightarrow+\infty} \frac{1}{n} \log \operatorname{Card}\{\gamma:|\gamma|=n\}
$$

i.e., $\exp (-h)$ is the radius of convergence of the generating function $G(z)$ for word length in $\pi_{1}(V)$.

(c) The asymptotic slope of $\mathcal{L}$ as $a \rightarrow \pm \infty$ is $-A$ and $-B$, respectively.

Proof. Parts (a) and (b) follow easily from standard properties of pressure [PP]. In particular, $h$ is the topological entropy of the subshift $\sigma: \Sigma_{A}^{+} \rightarrow \Sigma_{A}^{+}$.

For part (c) we recall that the slope of the curve $\mathcal{L}$ at $(a, F(a))$ is $F^{\prime}(a)=-\int_{\Sigma_{A}^{+}} r d \mu_{-a r}$, where $\mu_{-a r}$ is the Gibbs measure for $-a r[\mathrm{PP}]^{1}$ By the variational principle $[\mathrm{PP}]$ we have that

$$
h_{\mu_{-a r}}(\sigma)-a \int_{\Sigma_{A}^{+}} r d \mu_{-a r} \geq h_{\mu}(\sigma)-a \int_{\Sigma_{A}^{+}} r d \mu,
$$

${ }^{1} \mathrm{~A} \sigma$-invariant probability measure $\mu$ on $\Sigma_{A}^{+}$is called Gibbs if there exists a Hölder continuous function $g: \Sigma_{A}^{+} \rightarrow \mathbb{R}$ such that the pressure $P(g)$ satisfies

$$
P(g)=\sup _{\mu \in M\left(\Sigma_{A}^{+}\right)}\left(h_{\mu}(\sigma)+\int_{\Sigma_{A}^{+}} g d \mu\right)
$$

where the supremum is taken over the simplex $M$ of all $\sigma$-invariant Borel probability measures $\mu$ on $\Sigma_{A}^{+}$. 
for all $\sigma$-invariant probability measures $\mu$. Thus $\int_{\Sigma_{A}^{+}} r d \mu_{-a r} \leq \inf _{\mu} \int_{\Sigma_{A}^{+}} r d \mu+2 h / a$, where $h$ denotes the topological entropy of the subshift $\sigma: \Sigma_{A}^{+} \rightarrow \Sigma_{A}^{+}$. In particular, letting $a \rightarrow+\infty$, and using the weak density of probability measures supported on single periodic orbits for the subshift, we see that

$$
\lim _{a \rightarrow+\infty} F^{\prime}(a)=-\lim _{a \rightarrow+\infty} \int_{\Sigma_{A}^{+}} r d \mu_{-a r}=-\inf _{\mu} \int_{\Sigma_{A}^{+}} r d \mu=-\inf _{[\gamma] \in \pi_{1}(V)} l(\gamma) /|\gamma|=-A
$$

The proof of the second part of (c) is similar.

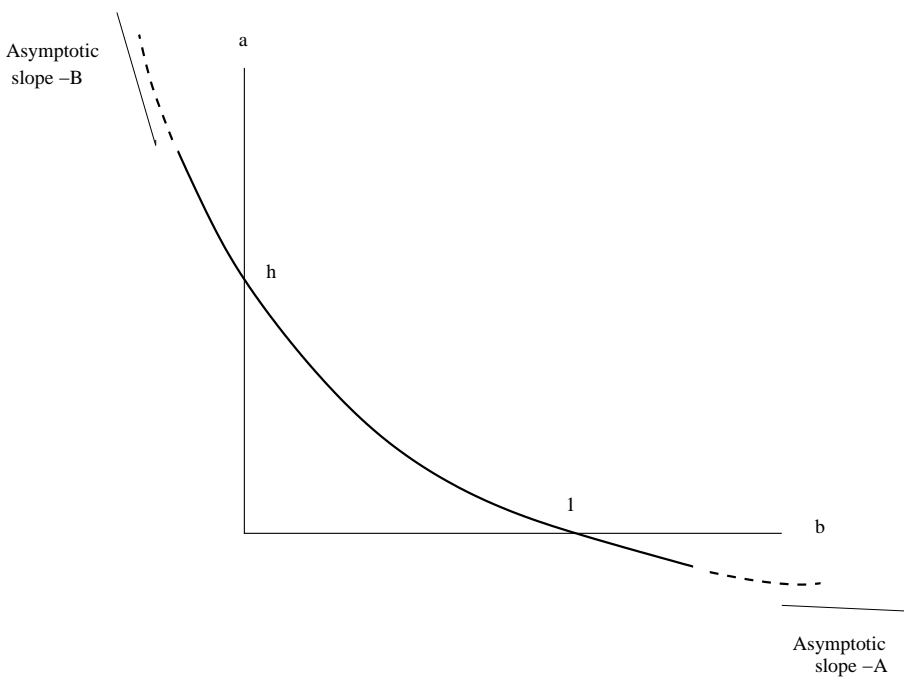

Figure 1. The Curve $\mathcal{L}$

\section{FREE ENERGY AS AN ISOMETRY INVARIANT}

Let $V_{1}$ and $V_{2}$ be negatively curved surfaces and let $\Gamma$ be a symmetric generating set for $\pi_{1}\left(V_{1}\right)$. If $\phi: V_{1} \rightarrow V_{2}$ is an isometry, then clearly the free energy of $V_{1}$ with respect to $\Gamma$ coincides with the free energy of $V_{2}$ with respect to the pushed forward generating set $\phi_{*} \Gamma$, i.e., $F_{\Gamma, V_{1}}=F_{\phi_{*} \Gamma, V_{2}}$.

Now suppose that $V_{1}$ and $V_{2}$ are negatively curved surfaces with generating sets $\Gamma_{1}$ of $\pi_{1}\left(V_{1}\right)$ and $\Gamma_{2}$ of $\pi_{1}\left(V_{2}\right)$, and assume that the free energies coincide, i.e., $F_{\Gamma_{1}, V_{1}}=F_{\Gamma_{2}, V_{2}}$. A natural rigidity question is whether this implies that the surfaces $V_{1}$ and $V_{2}$ are isometric? In this section we show that this is, in general, false. Thus in this sense, free energy is not a complete invariant of isometry.

Proposition 3. There exist two negatively curved hyperbolic surfaces $V_{1}$ and $V_{2}$ with generating sets $\Gamma_{1}$ of $\pi_{1}\left(V_{1}\right)$ and $\Gamma_{2}$ of $\pi_{1}\left(V_{2}\right)$ such that $F_{\Gamma_{1}, V_{1}}=F_{\Gamma_{2}, V_{2}}$, but $V_{1}$ and $V_{2}$ are nonisometric. 
Proof. We verify that one of the standard constructions of isospectral but nonisometric hyperbolic surfaces [Bus, page 304], with a suitable choice of generators for the fundamental group, provides a pair of nonisometric hyperbolic surfaces having the free energy.

We follow Buser's description almost verbatim. The building blocks for the construction are hyperbolic surfaces $\mathcal{B}$ of signature $(0,5)$ with boundary geodesics $\alpha_{1}, \alpha_{2}, \beta, \gamma_{1}$ and $\gamma_{2}$, satisfying $\ell\left(\alpha_{1}\right)=\ell\left(\alpha_{2}\right)<\ell(\beta)<\ell\left(\gamma_{1}\right)=\ell\left(\gamma_{2}\right)<\ell\left(\eta_{1}\right)<\ell\left(\eta_{2}\right) \leq 1$. See Figure 2. It follows from the collar lemma in hyperbolic geometry that the only isometry of such a five holed sphere is the identity. The two interior closed geodesics $\eta_{1}$ and $\eta_{2}$ decompose the $(0,5)$ surface into three $(0,3)$ surfaces (pairs of pants), which have been pasted together with a one-quarter twist.

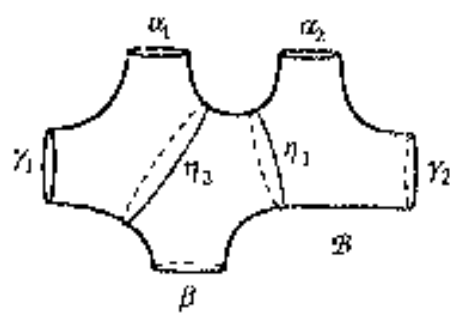

\section{Figure 2. The Building Block (From [Bus])}

We now glue together eight copies (building blocks) $\mathcal{B}_{0}, \ldots, \mathcal{B}_{7}$ of $\mathcal{B}$ with twist parameter zero according to the identifications shown in Figure 3 to obtain two compact hyperbolic surfaces $V_{1}$ and $V_{2}$. Buser proves that $V_{1}$ and $V_{2}$ are isospectral but not isomorphic. The proof that $V_{1}$ and $V_{2}$ are not isomorphic is a consequence of the above mentioned collar lemma.

We now construct a generating set for the fundamental group of $V_{1}$ and $V_{2}$. For, say $V_{1}$, choose all the boundary closed geodesics $\alpha_{1}, \alpha_{2}, \beta, \gamma_{1}$ and $\gamma_{2}$, as well as all the interior closed geodesics $\eta_{1}$ and $\eta_{2}$, for each of the eight building blocks. Several pairs of boundary closed geodesics are glued together to form $V_{1}$ and $V_{2}$, and it is easy to see that of the $8 \times 5=40$ boundary closed geodesics on the union of the eight building blocks, only 20 of these are distinct closed geodesics on $V_{1}$ and $V_{2}$. Since $V_{1}$ and $V_{2}$ have genus 13 , these 20 closed geodesics do not form a generating set. Since none of the interior closed geodesics are identified, all $8 \times 2=16$ are distinct closed geodesics on $V_{1}$ and $V_{2}$. We let $\Gamma$ denote the generating set of the fundamental group consisting of these $20+16=36$ generators. We claim that the free energies of $V_{1}$ and $V_{2}$ coincide with respect to the generating set $\Gamma$, i.e., $F_{\Gamma, V_{1}}=F_{\Gamma, V_{2}}$.

To see this, we exploit the very simple method of transplanting closed geodesics from $M_{1}$ to $M_{2}$, due to Buser and Bérard, and which is clearly explained in Section 11.5 of [Bus]. This provides a one-to-one length-preserving correspondence between the closed geodesics on both surfaces. In short, Buser decomposes a closed geodesic $c$ on $V_{1}$ into a sequence of disjoint subarcs $c_{j}$, with each subarc contained in one building block, and maps the 

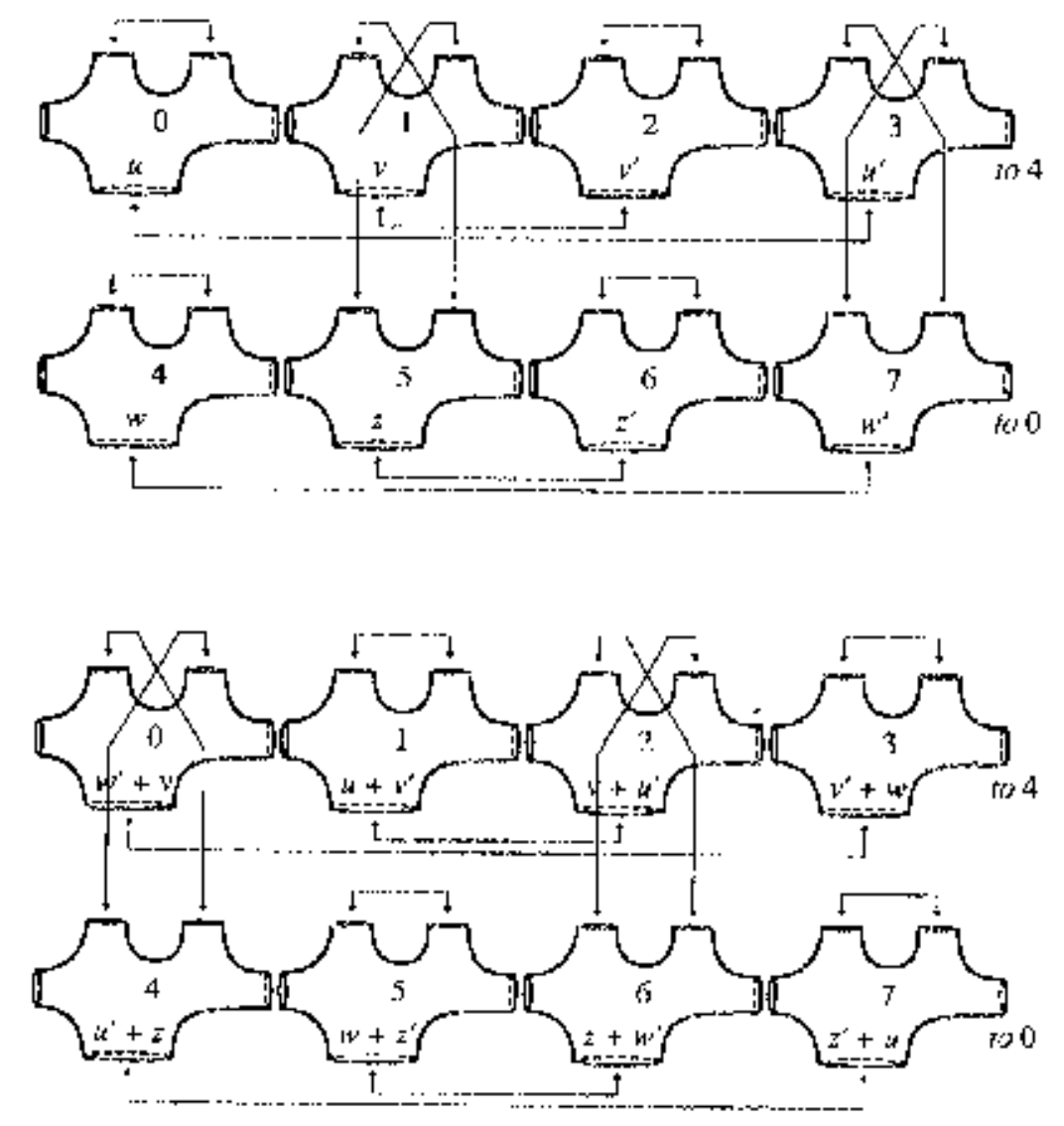

Figure 3. The surfaces $V_{1}$ And $V_{2}$ (From [Bus])

interior of each geodesic subarc $c_{j}$ to the interior of a geodesic subarc $c_{j}^{*}$ on $V_{2}$ using the family of identity mappings $\phi_{k k^{*}}$ from the interior of $\mathcal{B}_{k}$ to the interior of $\mathcal{B}_{k^{*}}$. Thus with the obvious interpretation, the transplantation mapping is the identity on the interiors of the eight building blocks. The very simple form of this correspondence between closed geodesics on the two surfaces is a consequence of the above mentioned fact that the only isometry of the building blocks is the identity.

It follows that every closed geodesic on $M_{1}$, which is not an element of $\Gamma$, and its transplanted closed geodesic on $M_{2}$, have the same length, and furthermore, have the same number of intersections with the closed geodesics in $\Gamma$. This completes the proof.

\section{The Rigidity of A NEW TYPE OF MARKED LENGTH SPECTRUM}

For a hyperbolic or negatively curved surface $V$, one defines the unmarked length spectrum of $V$ to be the set of lengths of all closed geodesics, i.e., $L_{V}=\left\{l(\gamma), \gamma \subset \pi_{1}(V)\right\}$. The marked length spectrum of $V$ consist of the lengths of closed geodesics, marked by the 
corresponding element of the fundamental group, i.e., the sequence $M_{V}=\{(l(\gamma),[\gamma]), \gamma \subset$ $\left.\pi_{1}(V)\right\}$. There is a significant amount of literature studying whether, or to what extent, the marked and unmarked length spectrum determine the metric [Bus, Cro, McK, Ota, Sun, Vig, Wol].

Motivated by our study of free energy, it is natural to study the length spectrum which is marked by the word length of the closed geodesic with respect to a fixed set of generators. This natural length spectrum lies between the well-known marked length spectrum and the unmarked length spectrum. More precisely, let $V$ be a compact negatively curved surface and let $\Gamma$ denote a set of generators for the fundamental group $\pi_{1}(V)$ of $V$. We define the $\Gamma$ word length marked length spectrum of $V$

$$
M_{\Gamma, V}=\left\{(l(\gamma),|\gamma|),[\gamma] \in \pi_{1}(V)\right\}
$$

Let $V_{1}$ and $V_{2}$ be negatively curved surfaces and let $\Gamma$ be a generating set for $\pi_{1}\left(V_{1}\right)$. If $\phi: V_{1} \rightarrow V_{2}$ is an isometry, then $M_{\Gamma, V_{1}}=M_{\phi_{*} \Gamma, V_{2}}$. Now suppose that $V_{1}$ and $V_{2}$ are negatively curved surfaces with generating sets $\Gamma_{1}$ of $\pi_{1}\left(V_{1}\right)$ and $\Gamma_{2}$ of $\pi_{1}\left(V_{2}\right)$, and assume that $M_{\Gamma_{1}, V_{1}}=M_{\Gamma_{2}, V_{2}}$. It immediately follows from definitions that $F_{\Gamma_{1}, V_{1}}=F_{\Gamma_{2}, V_{2}}$.

A natural rigidity question is whether $M_{\Gamma_{1}, V_{1}}=M_{\Gamma_{2}, V_{2}}$ implies that the surfaces $V_{1}$ and $V_{2}$ are isometric? The following proposition shows this is false.

Proposition 4. There exist two negatively curved hyperbolic surfaces $V_{1}$ and $V_{2}$ with generating sets $\Gamma_{1}$ of $\pi_{1}\left(V_{1}\right)$ and $\Gamma_{2}$ of $\pi_{1}\left(V_{2}\right)$ such that $M_{V_{1}, \Gamma_{1}}=M_{V_{2}, \Gamma_{2}}$, but $V_{1}$ and $V_{2}$ are not isometric.

Proof. The counterexample constructed in Section 2 also serves as a counterexample here. By construction, every closed geodesic on $V_{1}$ and its transplanted mate on $V_{2}$ have the same length and the same word length with respect to the generating sets $\Gamma_{1}$ and $\Gamma_{2}$ respectively.

\section{Asymptotic COMPARISON OF GeOdesic LENGTH AND WORD LENGTH}

For every unit tangent vector $v$ one can consider the associated geodesic with $\dot{c}(0)=v$. For any $T>0$, the geodesic arc $c([0, T])$ can be "closed up", by adding a second piece of geodesic arc of bounded length, to give a closed geodesic which we denote $\gamma_{v, T}$. If the limit $\lim _{T \rightarrow+\infty} l\left(\gamma_{v, T}\right) /\left|\gamma_{v, T}\right|$ exists, then it is easy to see that the limit $\alpha=\alpha(v)$ is independent of the particular construction of $\gamma_{v, T}$. The Birkhoff ergodic theorem implies that for almost all $v$ (with respect to the Liouville measure) the limit does exist and attains the constant value $\alpha_{0}$, where $A \leq \alpha_{0} \leq B$.

For $A \leq \alpha \leq B$ we define the dimension spectrum for geodesic length and word length

$$
f(\alpha)=\operatorname{dim}_{H}\left\{v \in T_{1} V: \lim _{T \rightarrow+\infty} \frac{l\left(\gamma_{v, T}\right)}{\left|\gamma_{v, T}\right|}=\alpha\right\},
$$

where $\operatorname{dim}_{H}$ denotes Hausdorff dimension [Fal]. The next result can be viewed as an asymptotic refinement of Milnor's result. 


\section{Proposition 5.}

(1) The dimension spectrum $f(\alpha)$ is an analytic function on $(A, B)$.

(2) For each $\alpha \in[A, B]$, the dimension spectrum $f(\alpha) \leq 3$, with equality if and only if $\alpha=\alpha_{0}$.

(3) For each $\alpha \in[A, B]$, the level set $\left\{v: \lim _{T \rightarrow+\infty} l\left(\gamma_{v, T}\right) /\left|\gamma_{v, T}\right|=\alpha\right\}$ is uncountable, dense, and supports an invariant Gibbs measure.

Proof. This is an immediate application of results on multifractal analysis in [Pes, PeW, Wei]. From the symbolic dynamics we can see that a unit tangent vector $v$, and its future orbit, correspond to an infinite word $x \in \Sigma_{A}^{+}$. Moreover, $l\left(\gamma_{v, T}\right) /\left|\gamma_{v, T}\right|$ converges to $\alpha$ if and only the Birkhoff average $S_{n} r(x) / n$ converges to $\alpha$. Since the roof function $r$ is not cohomologous to a constant, the multifractal analysis for the Birkhoff sum gives results corresponding to (1), (2), and (3) at the symbolic level, and using the above mentioned correspondence, these immediately translate into the geometric results claimed in the proposition.

\section{REFERENCES}

[AR] J. Anderson and A. Rocha, Analyticity of Hausdorff dimension of limit sets of Kleinian Groups, Ann. Acad. Sci. Fenn. Math. 22 (1997), 349-364.

[Bow] R Bowen, Symbolic Dynamics for Hyperbolic Flows, Amer. J. Math. 95 (1973), 429-460.

[BS] R. Bowen and C. Series, Markov Maps Associated with Fuchsian Groups, Inst. Hautes Etudes Sci. Publ. Math. 50 (1979), 153-170.

[Bus] P. Buser, Geometry and Spectra of Compact Riemann Surfaces, Birkhäuser, 1992.

[Can] J. Cannon, The Combinatorial Structure of Cocompact Discrete Hyperbolic Groups, Geom. Dedicata 16 (1984), 123-148.

[Eps] D. Epstein, Word Processing in Groups, Jones and Bartlett Publishers, 1992.

[Fal] K. Falconer, Fractal Geometry, Mathematical Foundations and Applications, Cambridge Univ. Press, 1990.

[McK] H. McKean, Selberg's Trace Formula as Applied to a Compact Riemann Surface, Comm. Pure Appl. Math. 25 (1972), 225-246.

[Mil] J. Milnor, A Note on Curvature and Fundamental Group, J. Diff. Geom. 2 (1968), 1-7.

[Ota] J. P. Otal, Le Spectre Marqué des Longueurs des Surfaces à Courbure Negative., Ann. of Math. 131 (1990), 151-162.

[PP] W. Parry and M. Pollicott, Zeta Functions and the Periodic Orbit Structures of Hyperbolic Dynamics, Astérisque 187-188 (1990).

[Pes] Y. Pesin, Dimension Theory in Dynamical Systems, Chicago Lectures in Mathematics, University of Chicago Press, Chicago, 1997.

[PeW] Y. Pesin and H. Weiss, The Multifractal Analysis of Gibbs Measures: Motivation, Mathematical Foundation, and Examples., Chaos 7 (1997), 89-106.

[PW] M. Pollicott and H. Weiss, Free Energy as a Dynamical Invariant (or Can You Hear the Shape of a potential?), To appear Comm. Math. Physics (2003).

[Rat] M. Ratner, Markov Decomposition for an U-flow on a Three-dimensional Manifold, Mat. Zametki 6 (1969), 693704.

[Rue] D. Ruelle, Thermodynamic Formalism, Addison-Wesley, 1978.

[Sim] B. Simon, The Statistical Mechanics of Lattice Gases, vol 1, Princeton U. Press, 1993.

[Sin] Y. Sinai, The Construction of Markov Partitions, Fun. ANal. Appl. 2 (1968), 70-80.

[Sun] T. Sunada, Riemannian Coverings and Isospectral Manifolds., Ann. of Math. 121 (1985), 69186. 
[TSK] T. Bedford, C. Series, M. S. Keane, Ergodic Theory, Symbolic Dynamics, and Hyperbolic Spaces, Oxford Science Publications, 1997.

[Vig] M. F. Vigneras, Variétés Riemanniennes Isospectrales et non Isometriques., Ann. of Math. 112 (1980), 21-32.

[Wei] H. Weiss, Spectrum of Equilibrium Measures for Conformal Expanding Maps and Axiom-A Surface Diffeomorphisms, JSP 95 (1999), 615-632.

[Wol] S. Wolpert, The Length Spectra as Moduli for Compact Riemann Surfaces., Ann. of Math. 109 (1979), 323-351. 